\title{
Postoperative Recovery of Light Sensitivity in Eyes with Rhegmatogenous Retinal Detachment
}

\author{
Tomohiro Ooshiro Hiroyuki lijima \\ Department of Ophthalmology, University of Yamanashi, Yamanashi, Japan
}

\section{Keywords}

Light sensitivity - Rhegmatogenous retinal detachment . Humphrey perimetry - Total deviation - Postoperative recovery

\begin{abstract}
Purpose: To investigate whether postoperative light sensitivity recovers completely to the level prior to the development of rhegmatogenous retinal detachment (RRD) after successful surgery. Methods: We retrospectively studied 44 eyes of 44 patients with RRD who were successfully operated and who underwent Humphrey central 30-2 perimetry postoperatively. The averaged total deviation in Humphrey perimetry in the reattached retina was compared with that of the horizontal or vertical counterpart in the preoperatively non-detached retina. Results: The averaged total deviation in the reattached retina was significantly lower than in its counterpart $(p<0.0001)$. The averaged residual loss of light sensitivity did not correlate with postoperative visual acuity ( $p=0.8047)$ or with its change $(p=0.1242)$. Conclusions: Light sensitivity in the detached retina in eyes with RRD does not completely recover after successful surgery.
\end{abstract}

(C) 2017 The Author(s)

Published by S. Karger AG, Basel

\section{Introduction}

In eyes with rhegmatogenous retinal detachment (RRD), especially those showing normal visual acuity without foveal detachment, the most common symptom is a visual field defect. This has generally been examined using Goldmann kinetic perimeter. However, this perimeter is more suited for detecting visual field constriction rather than visual field depression or reduction of light sensitivity because it measures the extent of isopters, or the "seeing limits," using test target lights of various sizes and intensities.

In comparison, static perimeters including Humphrey, OCTOPUS, and MP-1 microperimeter measure light sensitivity at multiple points within 5-30 degrees of the central visual field. Yet, it may be more advantageous to evaluate visual field depression in the posterior pole of the retina. As static perimetry measures light sensitivity at individual points in the posterior pole of the retina, serial tests of static perimetry may be used to investigate temporal changes of light sensitivity during disease progression or during recovery after retinal surgery [1-5].

While many previous reports have studied recovery of visual acuity following successful surgery to repair RRD eyes [6-10], few studies have investigated the re-

Hiroyuki Iijima

Department of Ophthalmology

University of Yamanashi

1110 Shimokato, Chuo, Yamanashi 409-3898 (Japan)

E-Mail hiijimar@yamanashi.ac.jp
E-Mail karger@karger.com www.karger.com/oph
(C) 2017 The Author(s)
Published by S. Karger AG, Basel

Karger

This article is licensed under the Creative Commons AttributionNonCommercial-NoDerivatives 4.0 International License (CC BYNC-ND) (http://www.karger.com/Services/OpenAccessLicense) Usage and distribution for commercial purposes as well as any 
Table 1. Demographic and clinical data of patients with rhegmatogenous retinal detachment

\begin{tabular}{lc}
\hline Number of eyes & 44 \\
$\quad$ Right & $16(36)$ \\
$\quad$ Left & $28(64)$ \\
Sex & $34(77)$ \\
$\quad$ Male & $10(23)$ \\
$\quad$ Female & $59.5(14-84)$ \\
Age, years & $7.5(0-87)$ \\
Preoperative days with retinal detachment & \\
Macular status & $30(68)$ \\
$\quad$ Macula off & $14(32)$ \\
$\quad$ Macula on & $0.398(-0.176$ to 2.301$)$ \\
Preoperative logMAR BCVA & $10(23)$ \\
Retinal surgery & $34(77)$ \\
$\quad$ Scleral buckling & $26(59)$ \\
$\quad$ PPV with or without PEA/IOL & $8(18)$ \\
$\quad$ With PEA/IOL & $97(8-547)$ \\
$\quad$ Without PEA/IOL & $0.126(-0.176$ to 0.699$)$ \\
Days between surgery and the first postoperative Humphrey perimetry & \\
Postoperative logMAR BCVA & \\
\hline
\end{tabular}

Data presented as $n(\%)$ or median (range). logMAR, logarithm of the minimal angle of resolution; BCVA, best-corrected visual acuity; PPV, pars plana vitrectomy; PEA/IOL, phacoemulsification and intraocular lens implantation.

covery of light sensitivity in the preoperatively detached and postoperatively reattached retina using static perimetry [11-15]. Functional recovery of the whole retina in postoperative RRD eyes may more accurately be assessed by a topographical measure of light sensitivity, rather than by visual acuity, which is a measure of visual function at the very center of the fovea. Therefore, we retrospectively analyzed the postoperative results of static perimetry in eyes following successful RRD surgery.

\section{Patients and Methods}

We studied postoperative Humphrey results retrospectively using an electronic medical record database of 44 patients who underwent successful RRD surgery with scleral buckling or pars plana vitrectomy (PPV) with or without phacoemulsification and intraocular lens implantation (PEA/IOL) at the University of Yamanashi Hospital.

Inclusion criteria were eyes with a preoperative demarcation line of retinal detachment crossing the macular area, which meant that the postoperative Humphrey perimetry result included both areas corresponding to the reattached retina and the non-detached retina. Reliable Humphrey 30-2 perimetry results with fixation loss scores less than $20 \%$, false positive errors less than $33 \%$, and false negative errors less than $33 \%$ obtained at any of the postoperative visits were used in the study. We excluded eyes with funduscopic evidence of glaucoma or retinal diseases which may influence perimetry results. Table 1 presents the subjects' demographic data.

Patients underwent complete ophthalmologic examinations, including measurement of best-corrected visual acuity (BCVA), slit-lamp examination, dilated fundus ophthalmoscopy, color fundus photography, and optical coherence tomography (OCT) using the Cirrus HD-OCT system (Carl Zeiss Meditec, Dublin, CA, USA). Differentiation between eyes with macula on and with macula off was confirmed by the OCT images. BCVA was measured as a decimal unit and converted to the logarithm of the minimal angle of resolution (logMAR) for statistical analyses. Humphrey perimetry central 30-2 results were recorded using the SITA Standard or SITA fast protocol (Humphrey Instruments, San Leandro, CA, USA) during one of the postoperative visits to examine recovery of visual function in addition to BCVA. The perimetry results were primarily used to provide the patients with information on their postoperative status.

As the study was not prospective in nature and as RRD surgery was scheduled as an emergency procedure, preoperative perimetry results were not obtained in most cases. However, 10 patients (age range: 16-67 years; median: 60 years) had undergone preoperative Humphrey testing. When multiple Humphrey results were available during the postoperative follow-up period, the oldest was used.

A grid of the total deviation values of the Humphrey results was superimposed on the preoperative fundus image that was inverted upside down and magnified in order to locate the blind spot on the optic nerve head (Fig. 1). We compared the averaged total deviation in the reattached retina with the horizontal or vertical counterpart in the preoperatively non-detached retina across either the vertical or horizontal midline, according to the preopera- 

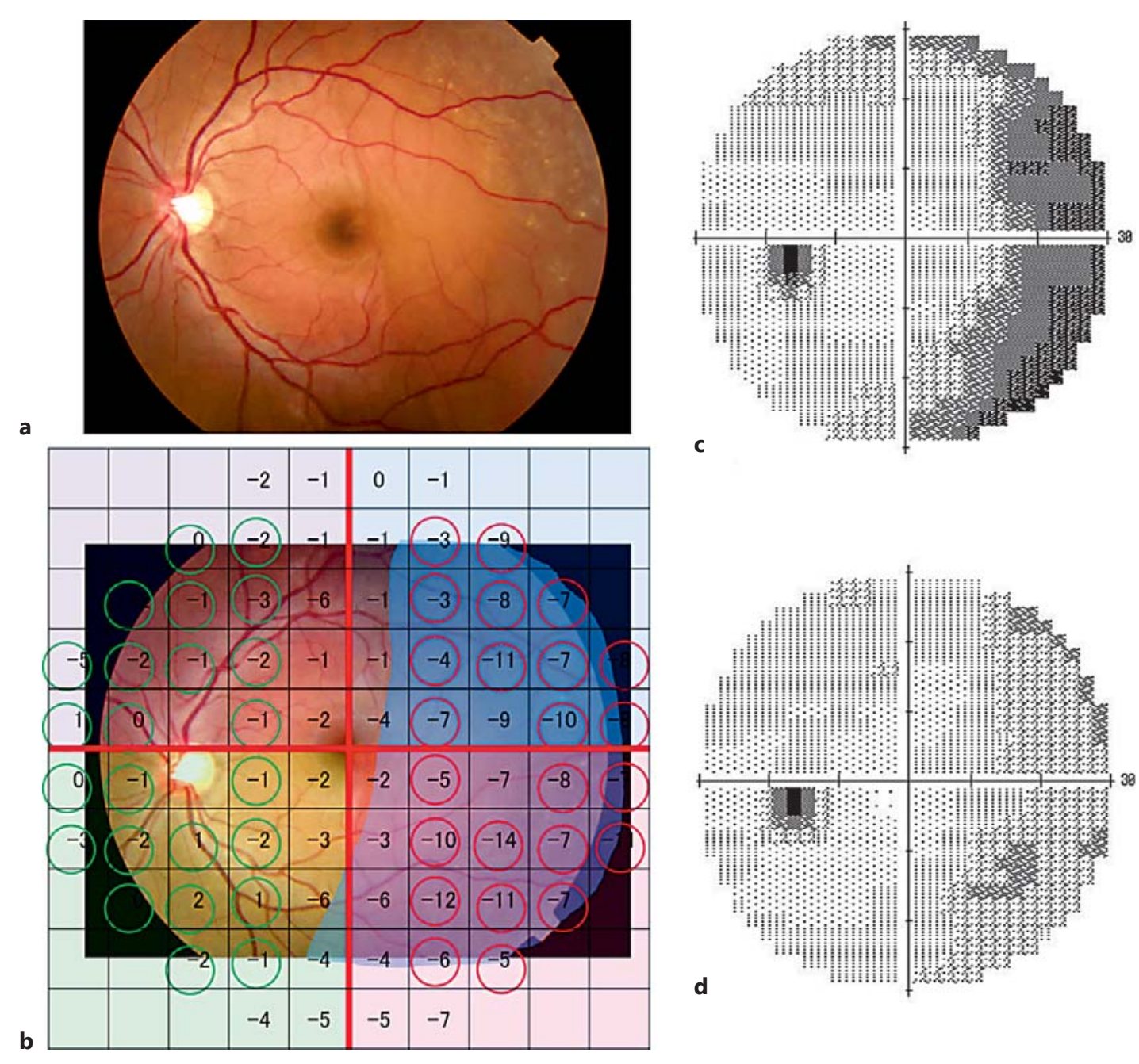

Fig. 1. Methods used to compare total deviation in the reattached retina with its counterpart in the preoperatively non-detached retina. a A preoperative fundus image of the left eye of a 26 -year-old male showing detachment in the temporal retina. $\mathbf{b}$ A grid of the total deviation values of the postoperative Humphrey 30-2 was superimposed over the preoperative fundus image, which is inverted upside down and magnified to locate the blind spot at the site of the optic nerve head. The red circles in the shaded area covering the preoperatively detached retina indicate the investigated points in the reattached retina that have normal counterparts in the preoperatively non-detached retina; these are indicated by green circles, opposite to the vertical midline in this case. c Grey scale display of the preoperative Humphrey 30-2. d Grey scale display of the postoperative Humphrey 30-2. tive pattern of retinal detachment. We did not include the data for the reattached retina when its counterpart was also detached preoperatively. Similarly, in the 10 selected cases, we compared the total deviation in the detached retina with its non-detached counterpart in the preoperative Humphrey results. The study was approved by the University of Yamanashi Hospital ethics committee and conformed to the Declaration of Helsinki. Statistical analyses were conducted using StatFlex (version 6.0, Artec Inc., Osaka, Japan). $p$ values less than 0.05 were considered to be statistically significant.

\section{Results}

The averaged total deviation in the reattached retina in all 44 cases was significantly lower than in its counterpart in the preoperatively non-detached retina at a symmetrical location across either the horizontal or vertical midline ( $p<0.0001$, Wilcoxon rank sum test; Fig. 2). The averaged total deviation was significantly lower in the reattached retina compared with that in its counterpart in the 


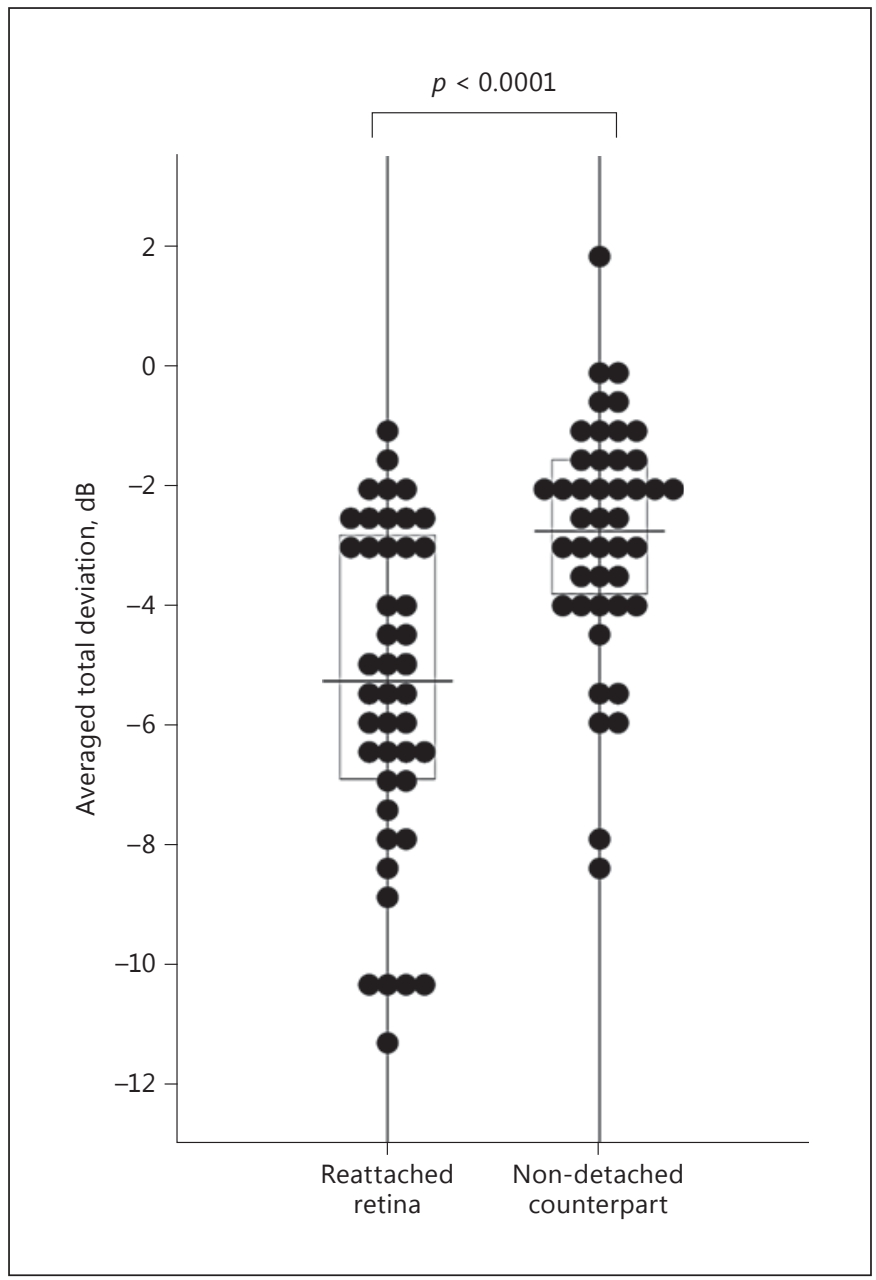

Fig. 2. Averaged total deviation in the reattached retina and in the preoperatively non-detached counterpart in the postoperative eyes. Horizontal bars indicate the median values and boxes indicate the 25 and $75 \%$ limits.

preoperatively non-detached retina in 36 eyes on which postoperative Humphrey perimetry was conducted at 30 days or later $(p<0.0001)$, and in 24 eyes at 90 days or later $(p<0.001)$.

Preoperative Humphrey results were available for 10 of the 44 subjects' eyes. Figure 3 shows the association between the averaged total deviation in the preoperatively detached retina and in the postoperatively reattached retina with its non-detached counterpart. Although light sensitivity in the reattached retina improved markedly after surgery, it appears sensitivity was still lower compared with that in its counterpart in the non-detached retina, which is considered to be equivalent to light sensitivity prior to development of retinal detachment in the reattached retina. Therefore, we defined the residual loss of

Postoperative Recovery of Light

Sensitivity in RRD

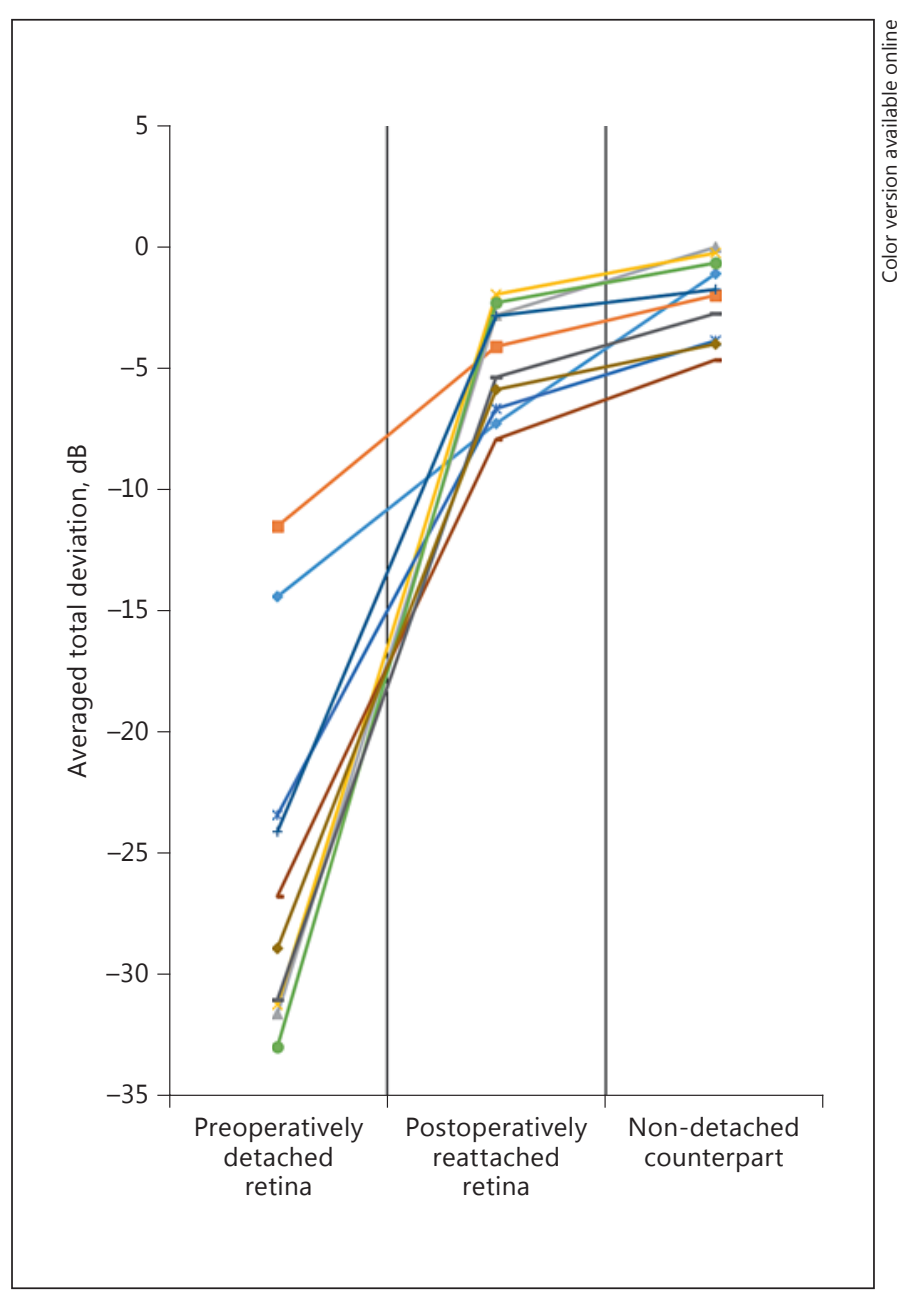

Fig. 3. Averaged total deviation in the preoperatively detached retina, the reattached retina, and the preoperatively non-detached counterpart in 10 selected eyes.

light sensitivity in the reattached retina as the difference between the total deviation in the reattached retina and in its non-detached counterpart.

The $\log$ MAR BCVA on the day of postoperative Humphrey testing decreased from the preoperative baseline in most eyes, indicating improvement of BCVA following surgery. The averaged residual loss of light sensitivity in each eye did not correlate with the change in logMAR BCVA (Spearman's correlation coefficient: $0.235, p=0.1242$; Fig. 4 ) or with the postoperative logMAR BCVA (Spearman's correlation coefficient: 0.038, $p=0.8047)$.

In 10 eyes following scleral buckling surgery, the mean of the residual loss of light sensitivity in each eye ranged between -7.67 and $-0.35 \mathrm{~dB}$, with a median of $-2.06 \mathrm{~dB}$. 


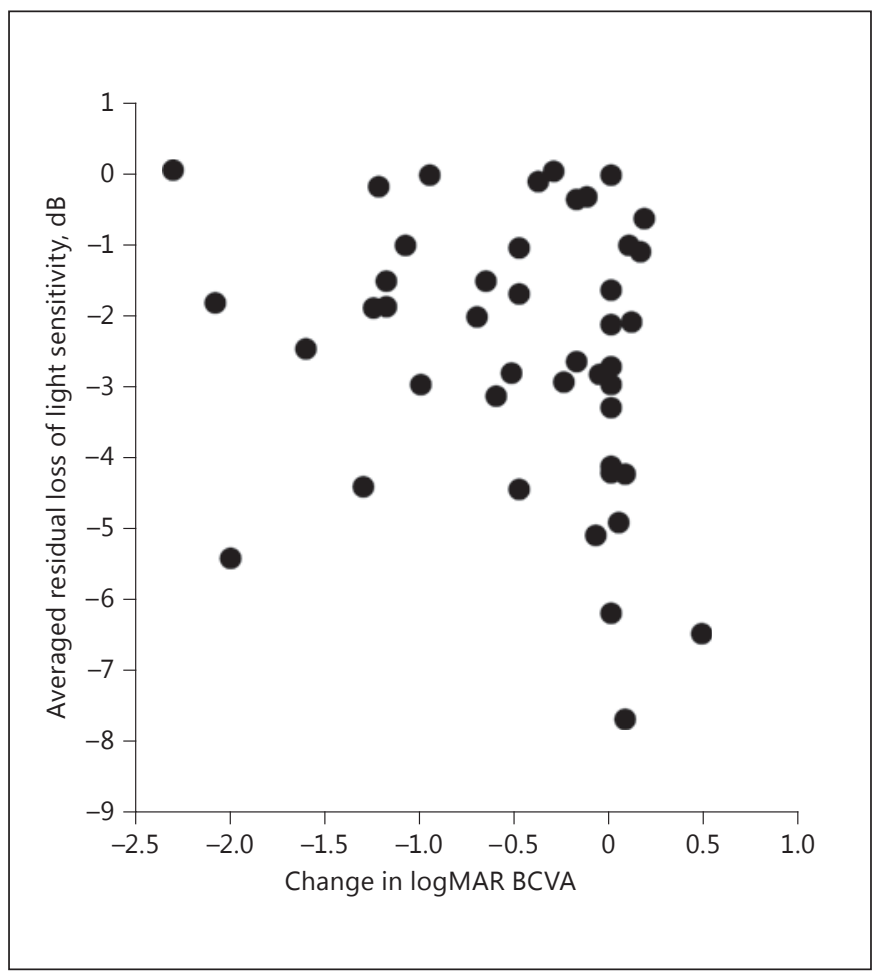

Fig. 4. Correlation between averaged residual loss of light sensitivity and change in logMAR BCVA. The averaged residual loss of light sensitivity is the mean difference between total deviation in the reattached retina and in the normal counterpart in each eye. $\log$ MAR, logarithm of the minimal angle of resolution; BCVA, best-corrected visual acuity.

In 34 eyes following PPV with or without PEA/IOL, means ranged between -6.46 and $0.06 \mathrm{~dB}$ with a median of $-2.26 \mathrm{~dB}$, which did not differ significantly from the scleral buckling surgery $(p=0.370$, Mann-Whitney $\mathrm{U}$ test).

The number of days between the onset of RRD, which was estimated using the history of visual symptoms including photopsia, floaters, or visual field defects, and the day of the surgery ranged from 0 to 87 days. The Spearman correlation coefficient for the averaged residual loss of light sensitivity and the number of days before surgery was 0.146 , which was not statistically significant ( $p=0.344$, Spearman's rank correlation test). The number of days between surgery and Humphrey perimetry testing ranged from 8 to 547 days. The Spearman correlation coefficient for the averaged residual loss of light sensitivity and the number of days between surgery and Humphrey testing was 0.062 , which was also not statistically significant ( $p=0.400$, Spearman's rank correlation test).

\section{Discussion}

RRD usually causes marked visual field defects, often with absolute scotoma corresponding to the detached retina meaning marked reduction of light sensitivity in the affected retina. A few studies have shown recovery of light sensitivity in the reattached retina after successful RRD surgery using static perimetry $[11,12]$. However, it is not known whether the reduced light sensitivity returns to the level of light sensitivity prior to disease development. In the current study we found that light sensitivity in the reattached retina greatly increased after surgery but was still less than light sensitivity in the non-detached counterpart, which is equivalent to the light sensitivity prior to the disease development in the reattached retina.

Accurate assessment of the recovery of light sensitivity in the reattached retina may require perimetric assessment before the development of RRD, which is not possible in most cases. Therefore, in the current study, we compared the total deviation obtained postoperatively between the reattached retina and its counterpart in the preoperatively non-detached retina opposite to the vertical or horizontal midline. We did so assuming that light sensitivity in the latter approximates the light sensitivity prior to disease development in the postoperatively reattached retina.

In the present study, the residual loss of light sensitivity in the reattached retina did not correlate with postoperative visual acuity or with changes in visual acuity. This implies that recovery of light sensitivity reflects functional recovery, which is different from visual acuity recovery [12]. Thus, to assess total recovery of visual function, assessing recovery of light sensitivity using static perimetry is important in addition to assessing visual acuity recovery.

Few studies have investigated the difference between scleral buckling surgery and vitrectomy in recovery of postoperative light sensitivity. In the present study, we found no significant differences in the recovery of light sensitivity after the two types of surgery. In eyes treated by vitrectomy and simultaneous PEA/IOL, increased light sensitivity in reattached retina may be partially attributed to the removal of the opaque lens. As we did not compare light sensitivity in the reattached retina with that in preoperative eyes but rather with its counterpart of the same postoperative eye, the results in the present study are unaffected by the removal of an opaque lens. The parameter of residual loss of light sensitivity, which is the difference in light sensitivity between the reattached retina and its counterpart in the preoperatively non-de-
56

Ophthalmologica 2017;238:52-58

DOI: $10.1159 / 000475476$
Ooshiro/Iijima 
tached retina in the same eye, could eliminate the effects of media opacity that cause reduction of light sensitivity in different retinal areas.

The incomplete recovery of light sensitivity in the reattached retina found in the present study may depend on the duration of retinal detachment prior to surgery. Using the static-perimetry mode of Goldmann perimetry from 1 to 8 years after successful surgery in 48 eyes with RRD, Zygulska-Mach et al. [15] found that reduced light sensitivity in the reattached retina was more pronounced in eyes with a longer duration of macular detachment. Isashiki and Ohba [12] studied the recovery of light sensitivity using Octopus perimeter in 16 eyes with RRD after scleral buckling surgery. They found that final recovery of light sensitivity correlated with the estimated duration of detachment, which ranged from 11 days to 22 months.

In the present study, we could not demonstrate a statistically significant association between the residual loss of light sensitivity in the reattached retina and the estimated duration of RRD. This may be due to the relatively short ( $<90$ days) preoperative duration of RRD in our subjects. Ozgur and Esgin [16] also did not find a correlation between the duration of macular detachment ranging from 3 to 51 days and the mean deviation of OCTOPUS perimeter in 29 postoperative eyes with macula-off RRD.

In addition, the present study did not find a statistically significant difference between the averaged residual loss of light sensitivity and the number of days before Humphrey testing. This may be due to the rapid recovery of light sensitivity postoperatively. Isashiki and Ohba [12] reported that recovery of light sensitivity appeared to reach a plateau 1-2 months after surgery.

The averaged total deviation in the preoperatively detached retina of 10 selected cases shown in Figure 3 was widely distributed, ranging from -33 to $-12 \mathrm{~dB}$. This indicates that the detached retina does not always result in absolute scotoma. Several eyes, especially those with shallow retinal detachment shown in Figure 1, tended to exhibit not absolute but relative scotoma in the corresponding visual field. Future studies with a larger sample size should investigate the association between the preoperative height of retinal detachment and light sensitivity reduction.

One limitation of our study was that it was retrospective in nature; thus, preoperative Humphrey data was not obtained in all cases. The number of days between postoperative Humphrey perimetry and surgery was also not controlled for. Because the postoperative Humphrey testing was the first experience for many of the subjects, the

Postoperative Recovery of Light

Sensitivity in RRD accuracy of the perimetric results was lowered because of absent learning effects. Moreover, clinical parameters including the height of the detached retina were not recorded precisely. Future prospective studies may therefore be necessary to clarify recovery of light sensitivity in postoperative eyes with RRD.

In conclusion, recovery of light sensitivity after successful RRD surgery does not achieve the level of its normal counterpart. This indicates incomplete recovery of light sensitivity in the reattached retina.

\section{Statement of Ethics}

Patient consent has been obtained. Ethics approval has been granted by the University of Yamanashi Hospital ethics committee.

\section{Disclosure Statement}

No competing interests are declared.

\section{Funding}

This study was supported in part by a JSPS KAKENHI Grant, number 25462707 (Grant-in-Aid for Scientific Research) from the Japan Society for the Promotion of Science (JSPS) (Hiroyuki Iiji$\mathrm{ma})$.

\section{Author Contributions}

T.O.: study conception, design, data acquisition. H.I.: data analysis, statistical analysis, drafting and revising of work.

\section{References}

1 Hirakawa H, Iijima H, Gohdo T, Imai M, Tsukahara S: Progression of defects in the central 10-degree visual field of patients with retinitis pigmentosa and choroideremia. Am J Ophthalmol 1999;127:436-442.

2 Imasawa M, Morimoto T, Iijima H: Recovery of visual field loss due to central retinal artery occlusion. Jpn J Ophthalmol 2004;48:294299.

3 Kumagai K, Furukawa M, Ogino N, Larson E, Uemura A: Long-term outcomes of macular hole surgery with triamcinolone acetonideassisted internal limiting membrane peeling. Retina 2007;27:1249-1254.

4 Sakai T, Iida K, Tanaka Y, Kohzaki K, Kitahara K: Evaluation of S-cone sensitivity in reattached macula following macula-off retinal detachment surgery. Jpn J Ophthalmol 2005; 49:301-305. 
5 Kremer I, Nissenkorn I, Lusky M, Yassur Y: Late visual field changes following cryotherapy for retinopathy of prematurity stage 3 . Br J Ophthalmol 1995;79:267-269.

6 Ross WH: Visual recovery after macula-off retinal detachment. Eye (Lond) 2002;16:440446.

7 Liu F, Meyer CH, Mennel S, Hoerle S, Kroll P: Visual recovery after scleral buckling surgery in macula-off rhegmatogenous retinal detachment. Ophthalmologica 2006;220:174180.

8 Schatz P, Andreasson S: Recovery of retinal function after recent-onset rhegmatogenous retinal detachment in relation to type of surgery. Retina 2010;30:152-159.

9 van de Put MA, Croonen D, Nolte IM, Japing WJ, Hooymans JM, Los LI: Postoperative re- covery of visual function after macula-off rhegmatogenous retinal detachment. PLoS One 2014;9:e99787.

10 Frings A, Markau N, Katz T, et al: Visual recovery after retinal detachment with maculaoff: is surgery within the first $72 \mathrm{~h}$ better than after? Br J Ophthalmol 2016;100:1466-1469.

11 Delolme MP, Dugas B, Nicot F, Muselier A, Bron AM, Creuzot-Garcher C: Anatomical and functional macular changes after rhegmatogenous retinal detachment with macula off. Am J Ophthalmol 2012;153:128-136.

12 Isashiki M, Ohba N: Recovery of differential light sensitivity following surgery for rhegmatogenous retinal detachment. Graefes Arch Clin Exp Ophthalmol 1986;224:184-190.

13 Lai WW, Leung GY, Chan CW, Yeung IY, Wong D: Simultaneous spectral domain OCT and fundus autofluorescence imaging of the macula and microperimetric correspondence after successful repair of rhegmatogenous retinal detachment. Br J Ophthalmol 2010;94: 311-318.

14 Rashid S, Pilli S, Chin EK, Zawadzki RJ, Werner JS, Park SS: Five-year follow-up of macular morphologic changes after rhegmatogenous retinal detachment repair: Fourier domain OCT findings. Retina 2013;33: 2049-2058.

15 Zygulska-Mach H, Starzycka M, Ciechanowska A: Retinal function after surgical treatment of detachment. Ophthalmologica 1979;178: 210-214.

16 Ozgur S, Esgin H: Macular function of successfully repaired macula-off retinal detachments. Retina 2007;27:358-364. 